\title{
History of Peripheral Vascular Disease
}

National Cancer Institute

\section{Source}

National Cancer Institute. History of Peripheral Vascular Disease. NCI Thesaurus. Code C66923.

An event in the personal medical history of peripheral vascular disease as evidenced by: 1 .

Claudication either with exertion or at rest; 2 . Amputation for arterial vascular insufficiency; 3. Aorto-iliac occlusive disease reconstruction, peripheral vascular bypass surgery, angioplasty or stent; or percutaneous intervention to the extremities; 4. Documented abdominal aortic aneurysm (AAA) repair or stent; 5. Positive noninvasive/invasive test. It does not include procedures such as vein stripping, carotid disease, or procedures originating above the diaphragm. (from NIH Roadmap Cardiovascular Data Standards Working Group) 